\title{
Fluorescein angiography in the differential diagnosis of sclerokeratitis
}

\author{
PETER WATSON AND SARAH BOOTH-MASON \\ From the Scleritis Clinic, Moorfields Eye Hospital, City Road, London EC1V 2PD
}

SUMMARY Anterior segment fluorescein angiography has been used in the investigation of patients with sclerokeratitis. This showed that corneal thinning or destruction was associated with nonperfusion of the episcleral vasculature. The changes arose either as a result of a systemic vasculitis in seropositive individuals or were induced by surgery to the eye. Infiltrative forms of sclerokeratitis were commoner in seronegative patients and were less often associated with vascular shutdown.

Destructive sclerokeratitis is a disastrous condition which can lead to loss of the eye through perforation. If it is treated vigorously and early, the prognosis is good, but an accurate diagnosis is vital if the correct treatment is to be given. Although some corneal change is found in $29 \%$ of patients with scleritis and $15 \%$ of patients with episcleritis, this becomes of importance only when the optical axis is obscured by opacities or when destructive changes occur. Early detection of the more serious forms of keratitis enables effective treatment to be instituted before the vision becomes affected. Anterior segment angiography has proved of considerable assistance in detecting the serious forms of this condition, and its efficacy as an aid to diagnosis is discussed in this paper.

The changes in the cornea in episcleritis are never severe and rarely permanent and result either from an extension of localised scleral oedema into the cornea, or to dellen formation caused by drying of an area of cornea adjacent to a region of intense episcleral oedema near the limbus.

Several distinctive patterns of corneal change are found in patients with scleritis. The changes are usually related to or in the same quadrant as the active scleral disease and may or may not be associated with vascularisation of the cornea.

Diffuse stromal keratitis is usually of rapid onset, occurring in the active stage of scleral inflammation. Superficial or mid stromal opacities appear within the corneal stroma and unless treated expand slowly, becoming surrounded by dense white 'precipitin Correspondence to P G Watson, FRCS, 17 Adams Road, Cambridge. ring'. If the reaction is intense, keratic precipitates may aggregate on the posterior corneal surface in the area of the stromal opacities.

Sclerosing keratitis may arise from a diffuse stromal keratitis which has failed to resolve completely or may arise de novo. The cornea changes to resemble sclera. The stromal opacifies containing lipid often looking like crystals of candy floss. The changes often start at the periphery but may advance towards the centre with alarming rapidity. If any vessels are present, they never occur at the advancing edge of the opacity.

Deep stromal keratitis is an unusual change in which a white opaque sheet of tissue invades the periperal cornea lying deep on Descemet's membrane. There may occasionally be an associated nummular corneal stromal opacity.

\section{LIMBAL GUTTERING AND KERATOLYSIS}

These are the most serious forms of corneal involvement in scleritis but present in various forms which need to be distinguished from each other.

(a) Limbal corneal thinning (contact lens cornea). The most benign form is one in which the peripheral cornea becomes thinned gradually over many years to resemble a contact lens on the cornea. All these patients have longstanding rheumatoid arthritis.

(b) Terriens-like changes. In this form the limbus becomes thinned within 2-3 mm of the limbal margin in the region of the active scleral disease. The region affected becomes opaque, vascularised, and occasionally extremely thin.

(c) Acute stromal keratitis and keratolysis. In very severe scleritis, areas of the central and peripheral 


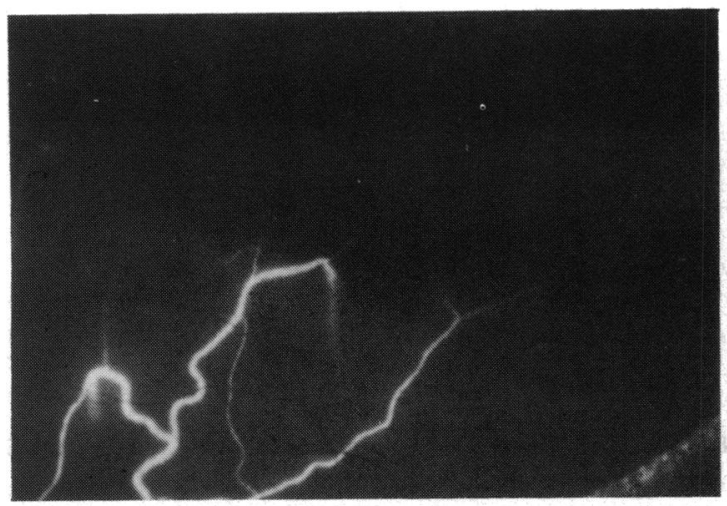

Fig. 1A

Figs. 1A, B, C Group 1A Anterior segment fluorescein angiogram from a patient who had rheumatoid arthritis and $a$ vascularised corneal gutter. (A) Early arterial phase. Large perforating vessels fill rapidly. (B) Early venous phase. The capillary network is patchily filled at the limbus only. The more proximal network is not perfused. The abnormal vessels in the cornea are filling from deep vessels arising deep to the sclera. (C) Late venous phase. There is still very poor filling of the capillaries at the limbus, and these vessels are grossly abnormal. There is deep and superficial leakage of dye. The proximal episcleral network is still unperfused.

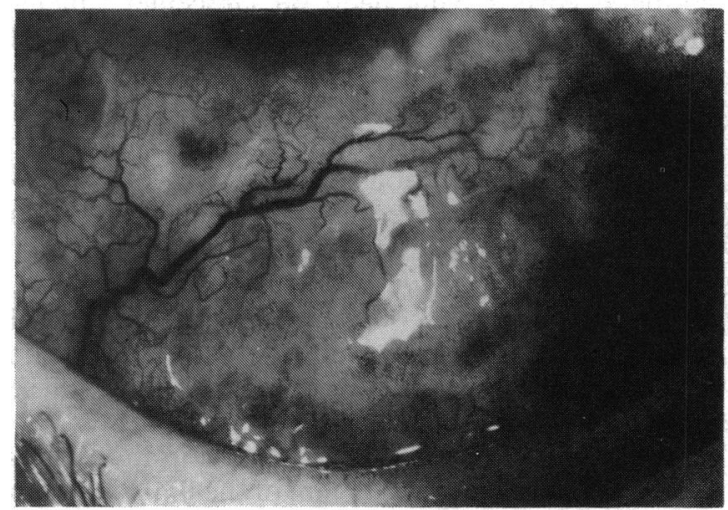

Fig. 2 Group 1A Corneoscleral necrosis in a patient with Wegener's granulomatosis. The destructive process not only involves both large areas of sclera but transgresses the limbus. Perirarteritis nodosa and Wegener's granulomatosis are the only conditions in which this occurs.

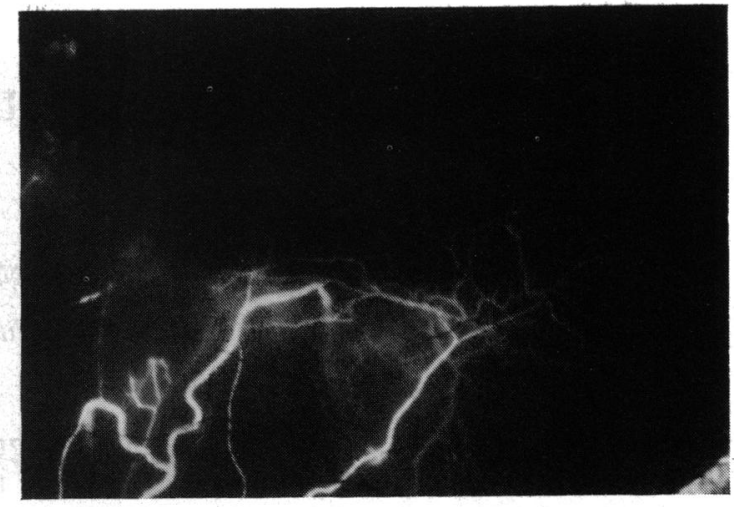

Fig. 1B

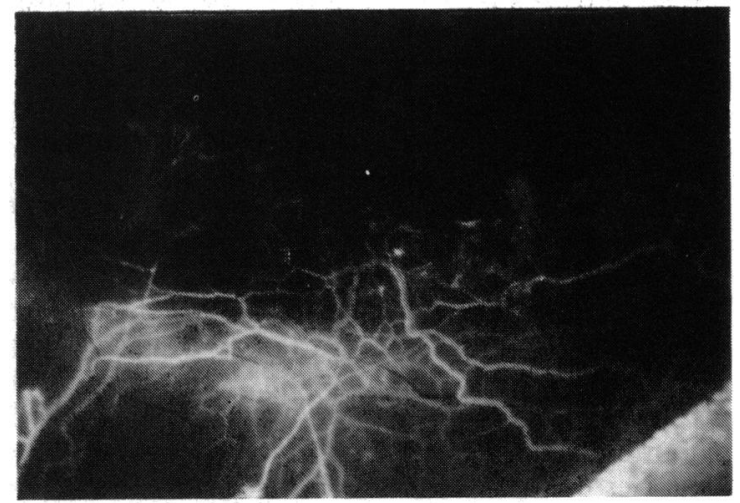

Fig. 1C

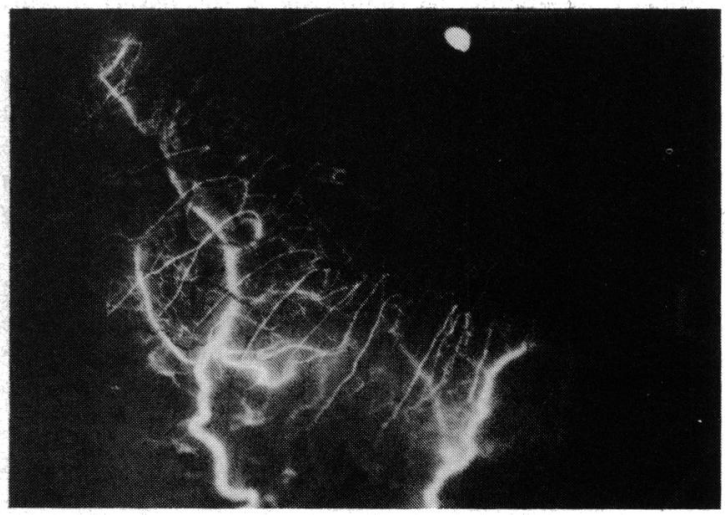

Fig. 3 Group 1A A similar appearance of the late venous phase to Fig. 1 in a patient who had an active sclerokeratitis but who had no overt connective tissue disease and had strong positive antinuclear and anti-DNA antibodies. Note the gross abnormality of the occluded capillaries. 


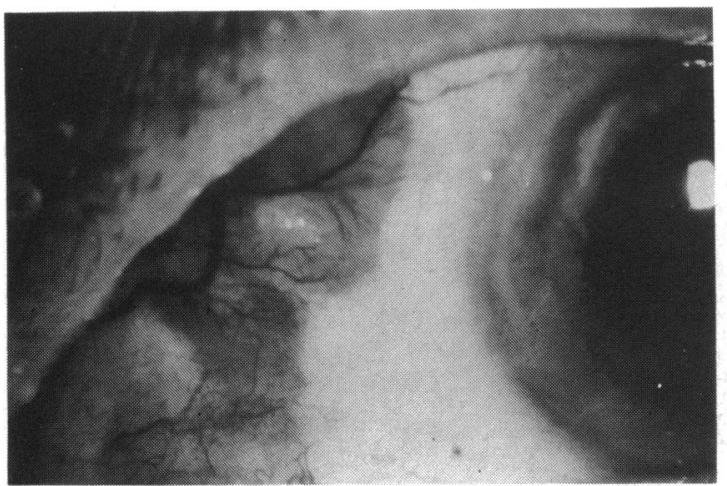

Fig. 4A

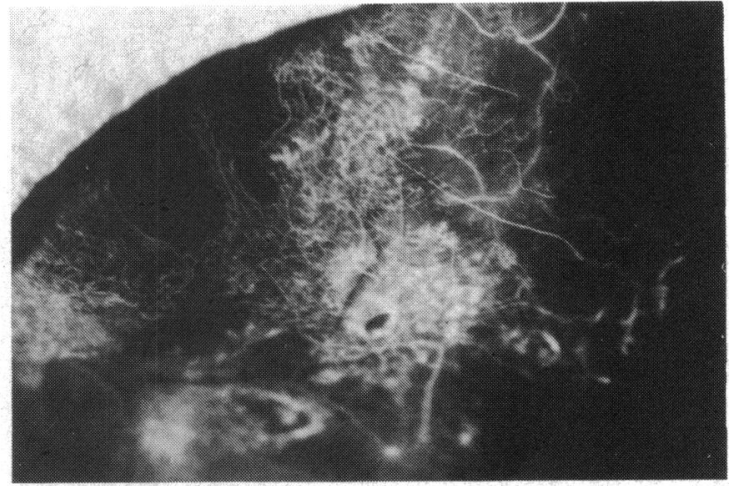

Fig. 4B

Figs. 4A, B Group 1B (A) Corneal gutter associated with a scleritis which followed extracapsular cataract extraction and posterior chamber lens implantation. The region adjacent to the limbus is white. In the area of intense scleral inflammation there are areas of scleral necrosis. (B) Anterior segment fluorescein angiogram at the limbus at the lower end of the gutter. Late venous phase. The gutter is vascularised from both superficial and deep vessels. There are some areas of conjunctival capillary dropout and some of the episcleral vessels do not fill. The whole vascular pattern is abnormal.

cornea opacify and swell. Initially the lesions are isolated, white or grey, but rapidly coalesce so that large areas of the cornea may become involved. Keratic precipitates are sometimes seen on the endothelium under the opacities whether a uveitis is present or not.

(d) Peripheral corneal melting. This condition starts as a grey, swollen, infiltrated area adjacent to an area of scleritis which rapidly breaks down, or, if there are several such areas, they coalesce to extend around the limbus. The gutter usually has a sharp well defined border on the limbal edge, but both the limbal and corneal margins may be undermined. Fine vessels extend to the edge of the ulcer, which in the early stages looks very like a Mooren's ulcer except

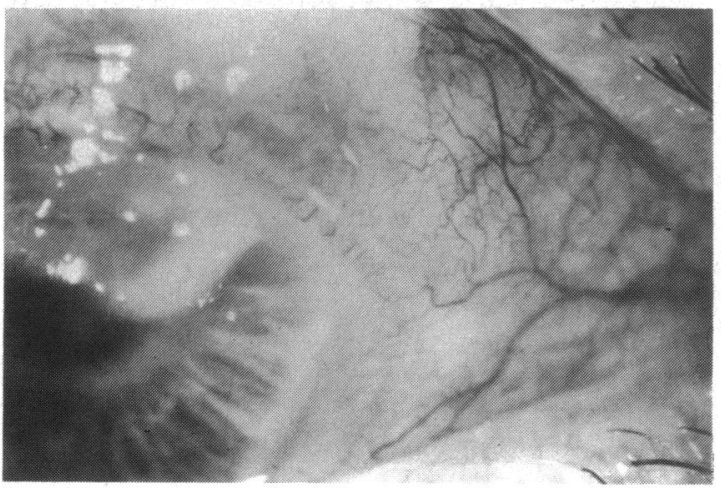

Fig. 5A for the intense scleral (as opposed to limbal) inflammation. Although this is the usual pattern in patients with scleromalacia perforans (necrotising scleritis without inflammation), the scleral inflammatory response is often very limited.

\section{Observations}

Forty-five patients who were attending the Scleritis Clinic at Moorfields Eye Hospital, City Road, London, and Addenbrooke's Hospital, Cambridge, were investigated. All had documented evidence of scleral and corneal disease and had had anterior segment fluorescein angiography performed.

Each patient was assessed for: (1) The type and

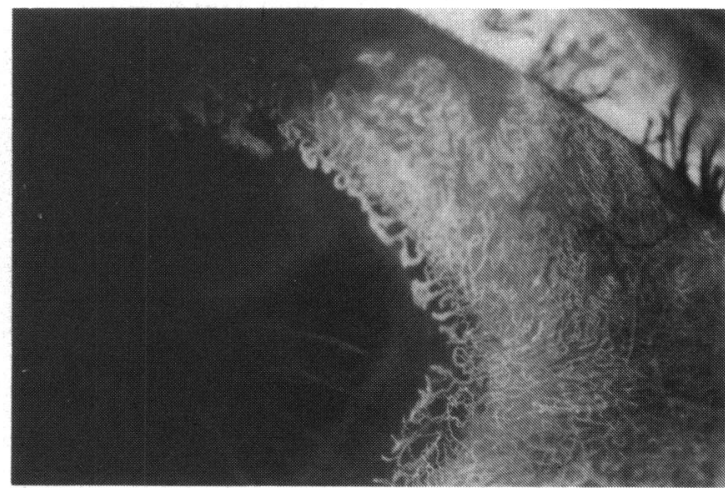

Fig. 5B

Figs. 5A, B Group 1B (A) Corneascleral necrosis following a trabeculectomy operation. (B) Anterior segment angiograph in the same region. Late venous phase. The conjunctival capillary network fills normally except at 12.00, where there is total absence of any perfusion. The upper and lower parts of the gutter are vascularised, and there is poor or absent filling of the episcleral circle deep to the conjunctival network. 




Fig. 6A

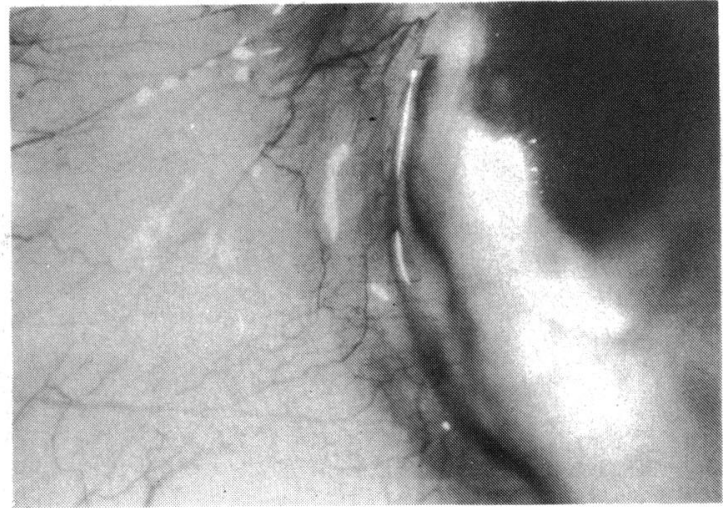

Fig. 6B

Figs. 6A, B Group 1B Healed corneal gutter following treatment in a patient who developed sclerokeratitis following intracapsular cataract extraction and Federov pupil clip lens implantation.

extent of scleritis. (2) The type and extent of the corneal changes. (3) The vasculature was documented by colour photographs and anterior segment fluorescein angiography. Particular attention was paid to the presence of vascular shutdown, flow patterns, and new vessel formation. (4) General condition: the presence of coexisting systemic disease or immunological evidence of this.

All the patients had scleral inflammation in one form or another but the patients were further divided into five groups according to the appearance of the corneal change and inspection of the anterior segment fluorescein angiograms (Table 1).

Group IA. Patients with corneal thinning, scleral inflammation, and vascular shutdown-idiopathic.

Group IB. Patients with corneal thinning, scleral inflammation, and shutdown-postsurgical.

Group II. Patients with corneal thinning, scleral inflammation, but with no vascular shutdown.

Group III. Patients with corneal infiltration, scleral inflammation, and vascular shutdown.

Group IV. Patients with corneal infiltration, scleral inflammation, but with no shutdown.

\section{Table 1 Patients in each group}

\begin{tabular}{llr}
\hline Group & Patients \\
\hline IA & $\left.\begin{array}{l}\text { Corneal thinning } \\
\text { Vascular shutdown }\end{array}\right\}$ & idiopathic \\
IB $\quad \begin{array}{l}\text { Corneal thinning } \\
\text { Vascular shutdown }\end{array}$ & postsurgical & 11 \\
II $\quad \begin{array}{l}\text { Corneal thinning } \\
\text { No vascular shutdown } \\
\text { III }\end{array}$ & $\begin{array}{l}\text { Corneal infiltration } \\
\text { Vascular shutdown }\end{array}$ \\
IV $\quad \begin{array}{l}\text { Corneal infiltration } \\
\text { Noshutdown }\end{array}$ & 2 \\
\hline
\end{tabular}

\section{GROUPS IA AND IB}

There were 11 patients in group IA (23.9\%) and eight in group IB $(17 \cdot 37 \%)$. Fluorescein in angiography in both groups revealed large areas of capillary shutdown both adjacent to and often away from the region of the corneal gutter. Figs. 1-8.

The eight patients in group IB all developed sclerokeratitis following surgery to the eye. Three followed routine intracapsular cataract extraction; one followed routine extracapsular cataract extraction with implant; one followed bilateral intracapsular cataract extraction but the affected eye had a leaking bleb; one followed a trabeculectomy done one month after a combined cataract and trabeculectomy in the fellow eye; one followed revisioning of an established trabeculectomy bleb; one followed a routine peripheral iridectomy. A localising cause was

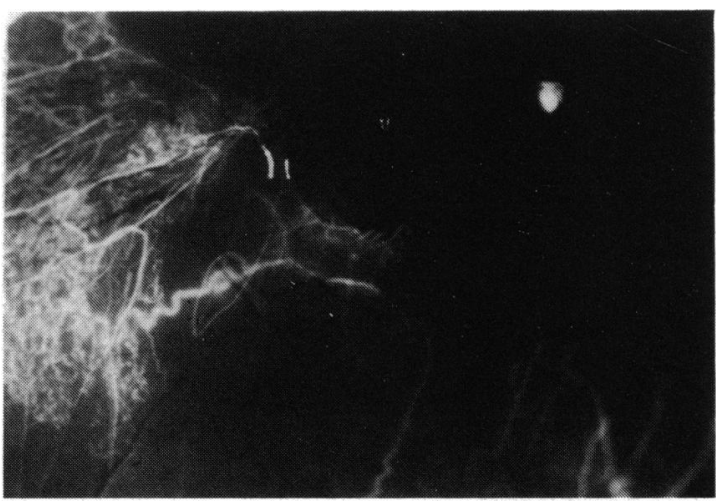

Fig.7 Group 1B Anterior segment fluorescein angiogram at the lower end of the gutter in a patient with a similar presentation to Fig. 6 which followed a simple, uncomplicated intracapsular extraction of cataract. There is a total occlusion of vascular network beyond the extent of gutter. All the visible vessels here are new vessels. 


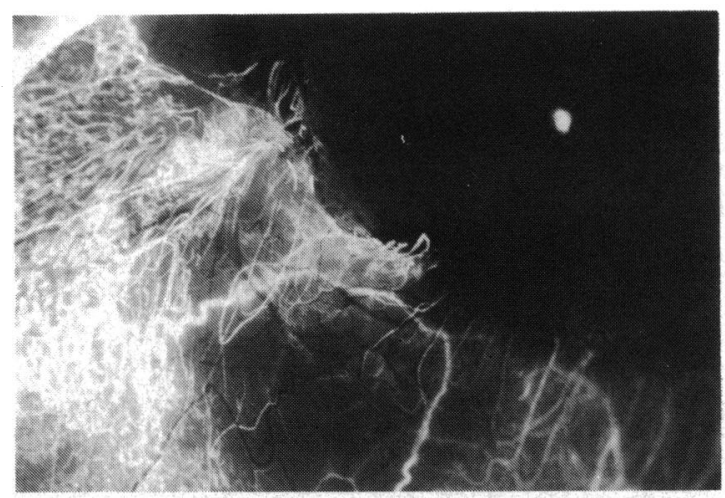

Fig. 8 Group 1B Anterior segment fluorescein angiogram in the same patient as Fig. 7 taken three months later after treatment and in a quiescent stage. There is still no perfusion of the region beyond the gutter even at this late stage in the angiogram. It appears that once vessels have been occluded in this situation they do not recanalise.

found in only one patient in group IA (the scleritis starting at a site of infection), but $83 \%$ of these patients had evidence of connective tissue disease.

Manifest connective tissue disease was present in six patients in the two groups $(31.57 \%)$. Of these, three patients had rheumatoid arthritis (RA); three patients had Wegener's syndrome (one of whom had RA as well); five patients were in group IA; one patient was in group IB. All these patients with Wegener's granulomatosis had necrotising disease and two of the three patients with RA had necrotising disease.

Immunological changes. Ten of 11 patients in group IA had serological evidence of neurological abnormalities $(90 \%)$; five of eight patients in group IB had abnormalities $(62.5 \%)$; three patients had no serological immunological abnormality; one patient was in group IA and had severe RA and therefore can be presumed seropositive and has been included above; one patient was healthy in group IB and was probably normal. There were four patients with no serological abnormality ( $21 \%$ of both groups): three were in group IB; one was in group IA, and this was the patient whose lesions had an infective aetiology.

Uveitis. Cells and occasionally flare in the anterior chamber were noted during active stages of the disorder in seven patients $(36 \cdot 84 \%)$, but a definite attack of uveitis with synechia formation and keratic precipitates was documented in only four patients ( $21 \%$ of total). Three of these patients were in group IB. None of these patients with uveitis were seropositive (rheumatoid factor or antinuclear antibodies).

Of the types of destructive change found:

(1) Peripheral corneal melting - i.e., undermining

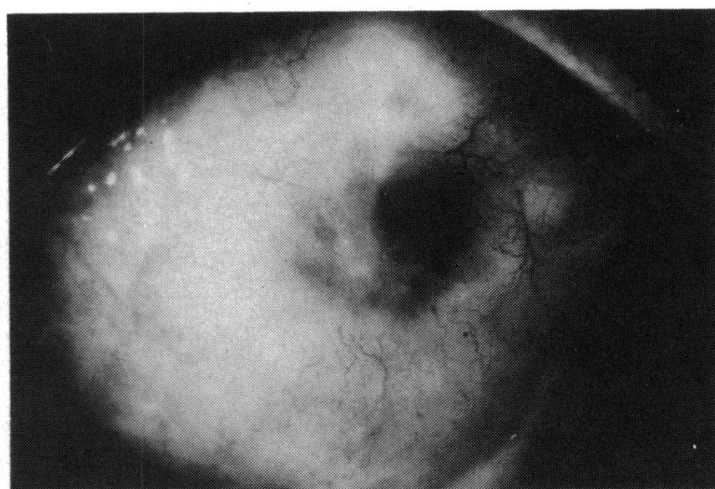

Fig. 9 Group III Sclerosing keratitis in a man with longstanding diffuse anterior scleritis.

of corneal edge, occurred in five patients in group IA and four patients in group IB.

(2) Terriens-like-i.e., sharply defined corneal edge occurred in five patients in group IA and four patients in group IB.

(3) Keratolysis-i.e., central and corneal thinning with or without peripheral changes occurred in one patient in group IA.

Corneal vascularisation was present in eight patients in group IA and six patients in group IB (i.e., total of $73.68 \%$ ).

Of the scleritis present, in group IA four patients had diffuse scleritis $(36 \cdot 36 \%)$ and seven patients had necrotising scleritis $(63.6 \%)$. In group IB two patients had diffuse scleritis $(12 \%)$, one patient had nodular scleritis $(25 \%)$, and five patients had necrotising scleritis $(62 \cdot 5 \%)$.

GROUP II

Corneal gutter scleral inflammation, no vascular shutdown. Only two patients fulfilled the criteria, and, of these, one showed gross vascular anomalies but no perfusion problems.

\section{GROU P III}

Nine patients fell into this category (19.5\%) (Figs. 9-11).

Corneal changes. All the patients showed an immune response, that is, a precipitin ring either at the edge of the lesion or spreading into the cornea. A variety of other non-specific changes were also noted, such as lipid deposition, limbal infiltrates, and oedema.

Corneal vascularisation was present in all patients $(100 \%)$.

Of the scleritis present four patients had diffuse $(44 \%)$, three had nodular $(36 \cdot 33 \%)$, and two had necrotising $(22 \%)$ scleritis.

Manifest connective tissue disease. No patient 


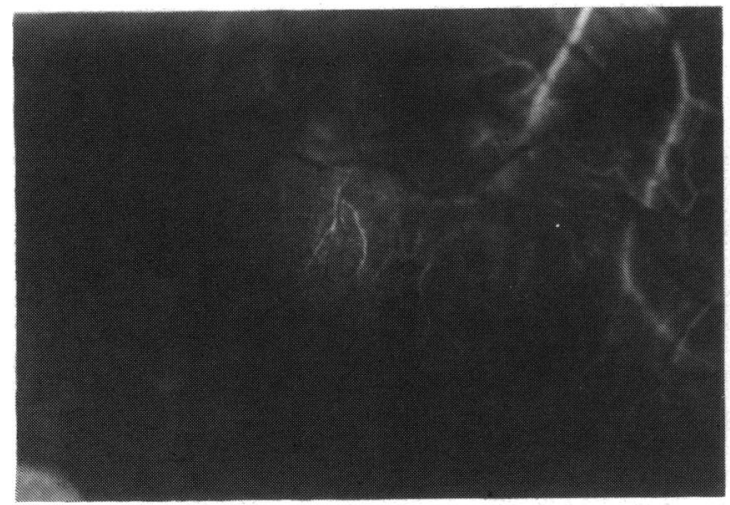

Fig. 10A



Fig. 10B

Fig. 10A, B Group III A patient with a similar corneal appearance to Fig. 9. The deep perforating arteries fill fast and normally, but the deep episcleral plexus fails to fill. The sclerosing keratitis at the limbus contains numerous new vessels.

in this group had any manifest connective tissue disorder.

Immunological changes were present in eight patients $(88 \cdot 8 \%)$.

Uveitis. One patient had definite uveitis and was seronegative $(11 \cdot 11 \%)$.

\section{GROUP IV}

Corneal infiltration, scleral inflammation, no shutdown in angiography: there were 15 patients in this group $(32 \cdot 6 \%)$.

Corneal vascularisation. Only eight patients showed the presence of vessels $(53 \cdot 3 \%)$.

Scleritis present. Twelve patients had diffuse $(80 \%)$ and three had nodular $(20 \%)$ scleritis (one with early necrosis).

Immunological changes. Twelve patients showed some changes, but in a few this was evident only during disease activity $(80 \%)$.

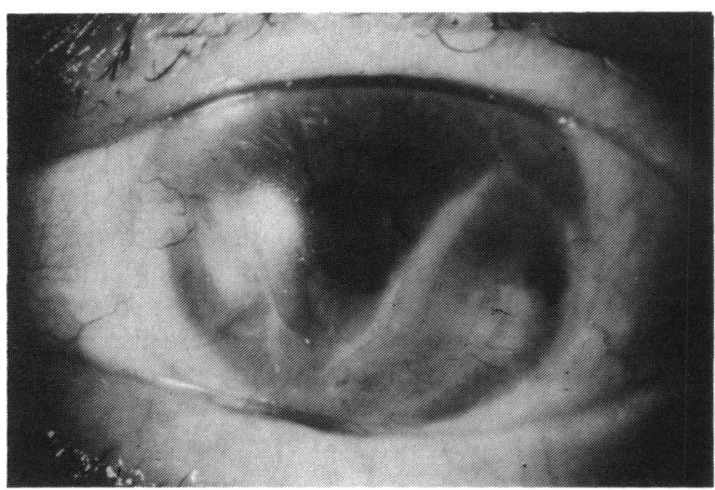

Fig. 11A
Manifest connective tissue disease was present in only three patients ( $20 \%)$ : one had RA and Sjögrens syndrome, one had ulcerative colitis (UC), one had Crohn's disease and arthritis (one patient also had congenital syphilis and one patient had orbital Wegener's granulomatosis).

Uveitis. A uveal reaction was reported in 11 patients but a definite uveitis documented in seven patients $(46 \cdot 66 \%)$. Five of these patients were seronegative $(71 \cdot 4 \%)$.

\section{Discussion}

Anterior segment fluorescein angiography has been found to be helpful in scleritis to differentiate patients who have vascular occlusive changes ${ }^{1}$ requiring intensive systemic steroid therapy for its control from those who have normal vascular patterns and who respond well to non-steroidal anti-inflammatory

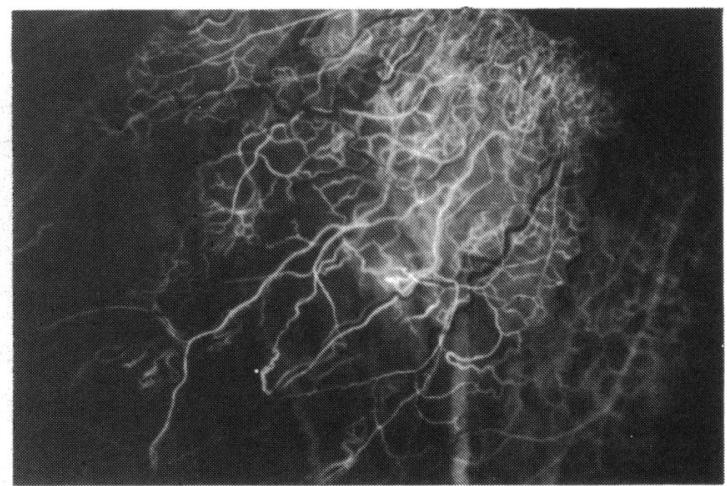

Fig. 11B

Figs. 11A, B Group IV Lipid infiltration at the limbus following recurrent attacks of sclerokeratitis. (A) Anterior segment fluorescein angiogram at 8.30. The limbus is normally perfused at a very high rate of flow; the whole vascular network being filled within 3 seconds of the appearance of the dye. 
therapy. This paper evaluates the use of this technique in the investigation of those who, in addition to scleral inflammation, also have corneal changes.

Corneal changes are observed in approximately one-third of those patients who have scleral disease. It has been known for many years that there are two major types of corneal change in scleral disease, namely, that in which the cornea becomes opaque and vascularised and that in which various forms of absorptive change of the cornea exist. This absorption can vary from an aggressive infiltrative and destructive keratolysis involving the peripheral cornea or complete corneal melt to a slowly progressive thinning of the peripheral cornea which may take many years.

Examining both the corneal changes and the fluorescein angiograms which were taken during the course of the disease, we found that five almost distinct groups could be identified depending on the type of corneal change (infiltration or thinning) and whether or not there was evidence of vasoobliteration of the episcleral or limbal vessels. In the group in which there was both vascular shutdown and corneal thinning two further subgroups emerged, because it became obvious that the corneal changes had been precipitated by surgery to the eye. Immunological examination of these patients revealed that $60 \%$ of them had some immunological abnormality, though only one had manifest connective tissue disease (rheumatoid arthritis). By contrast, $90 \%$ of the other group (so called idiopathic) had serological abnormalities, and $45 \%$ of them had manifest seropositive connective tissue disease (rheumatoid arthritis, Wegener's granulomatosis, or periarteritis nodosa). There was no difference in the types of corneal thinning or vascularisation or scleral disease between the two groups. All these patients with Wegener's granulomatosis and two of the three patients with rheumatoid arthritis had necrotising disease. Two of the three patients with Wegener's granulomatosis and one with polyarteritis nodosa showed destructive changes which transgressed the limbus.

It would seem therefore in this group that both types have the same characteristics in that there is a 'vasculitis' of the episcleral and limbal vessels which leads to vascular shutdown which in turn leads to catabolic resorption of the stromal tissue. If the systemic disease is severe, then the destructive changes occur spontaneously or, as in the case of one of these patients, as a result of intercurrent infection.

Patients who have a systemic vasculitis but with an apparently normal eye can be shown to have areas of vascular occlusion or non-perfusion. If such a patient had an eye operation, or if there were circulating immune complexes, these would be precipitated at the site of surgery and an autoimmune response could be induced. In the postoperative group of patients $60 \%$ had some serological evidence of immunological abnormality, and such a mechanism might be the cause of the corneal destruction. It is noticeable that those who have problems after surgery tend to develop a scleritis if the sclera is disturbed by surgery (trabeculectomy or retinal detachment) and a corneal thinning if they have a cataract extraction with corneal section. The part that any intercurrent infection plays in this process is unclear, and it was not possible to detect any obvious infection in this postoperative series, but the notes almost always indicated that the eye had been continuously irritable following surgery. Uveitis occurred only in the one seronegative patient.

It was extremely unusual to find corneal thinning or melting without vascular shutdown (group II), and one of the two patients in this group was later known to have developed shutdown. The other patient, however, seemed to be similar to the patients in group I, so this change remains unexplained.

By contrast, even though the scleral disease was severe, vascular occlusive changes were much less common in patients who developed corneal infiltration as opposed to corneal thinning and the vasoobliterative changes were much more patchy. Eight of the nine patients who showed shutdown had immunological abnormalities, but none had manifest connective tissue disease. Again uveitis occurred only in the seronegative patients. Necrotising disease is, as would be expected, rare in this situation.

The group who showed corneal infiltration without vascular changes also contained the largest number of patients with seronegative arthritis and with uveitis, which again occurred only in seronegative patients.

It would appear therefore that scleral thinning or destruction occurs when there is obliteration of the episcleral network of vessels. The series indicates that this is a result of an immunologically induced vasculitis regardless of whether the patient has manifest connective tissue disease or not. The other forms of corneal infiltration, though often the result of a local immune response, are not necessarily associated with permanent vascular occlusion and are much more likely to occur in seronegative patients. Uveitis in patients with sclerokeratitis has been found only in seronegative patients.

\section{References}

1 Watson PG, Bovey E. Anterior segment fluorescein angiography in the diagnosis of scleral inflammation. Ophthalmology (Rochester) 1985; 92: 1-11.

2 Watson PG, Hazleman BL. The sclera and systemic disorders. London and Philadelphia: Saunders, 1976. 